\title{
Performance Characteristics of Discrete- Time Queue With Variant Working Vacations
}

\author{
P. Vijaya Laxmi, Department of Applied Mathematics, Andhra University, Visakhapatnam, India \\ Rajesh P., Department of Mathematics, Vignan's Institute of Information Technology, Visakhapatnam, India
}

\begin{abstract}
This article analyzes an infinite buffer discrete-time single server queueing system with variant working vacations in which customers arrive according to a geometric process. As soon as the system becomes empty, the server takes working vacations. The server will take a maximum number K of working vacations until either he finds at least on customer in the queue or the server has exhaustively taken all the vacations. The service times during regular busy period, working vacation period and vacation times are assumed to be geometrically distributed. The probability generating function of the steady-state probabilities and the closed form expressions of the system size when the server is in different states have been derived. In addition, some other performance measures, their monotonicity with respect to $\mathrm{K}$ and a cost model are presented to determine the optimal service rate during working vacation.
\end{abstract}

\section{KEYWORDS}

Geometric Distribution, Probability Generating Function, Queue, Variant Working Vacations

\section{INTRODUCTION}

Unlike the classical vacation queues where the server suspends the services temporarily, in working vacation (WV) queues the server is active during the vacation period which is called working vacation (WV), Servi and Finn (2002). Wu and Takagi (2006) generalized Servi and Finn's (2002) M/M/l/ $W V$ queue to an $M / G / 1 / W V$ queue. Banik et al. (2007) studied a general input $G I / M / 1 / N / W V$ queue. The stochastic decomposition results of an $M / M / 1$ queue with WV have been derived by Liu et al. (2007) and the corresponding M/G/1 queue was studied by Li et al. (2009).

The analysis of discrete time queueing models has received considerable attention in view of their application in practical problems that arise from communication and computer systems including time-division multiple access (TDMA) schemes, asynchronous transfer mode (ATM), multiplexers in the broadband integrated services digital network (B-ISDN), management in service system and electronic commerce, etc. Past work on discrete-time queues is found in Meisling (1958), Hunter (1983), Takagi (1993). Tian and Zhang (2002), Alfa (2003). Li and Tian (2007) considered a discretetime GI/Geo/1 queue with WV and vacation interruption. Li et al. (2007) considered a discrete-time GI/Geo/1 queue with MWV under Early Arrival System (EAS) and Late Arrival System (LAS) schemes. Li and Tian (2008) and Tian et al. (2008) have analyzed a Geo/Geo/1 queue with single working vacation (SWV) and multiple working vacation (MWV), respectively. A discrete-time renewal input finite buffer batch service queue with MWVs has been studied by Vijaya Laxmi and Jyothsna (2014) using the supplementary variable techniqeue and the corresponding queue with balking and SWV has been presented by Vijaya Laxmi et al. (2015). Recently, a retrial queue with working vacation

This article, originally published under IGI Global's copyright on April 1, 2020 will proceed with publication as an Open Access article starting on February 1, 2021 in the gold Open Access journal, International Journal of Operations Research and Information Systems (converted to gold Open Access January 1, 2021), and will be distributed under the terms of the Creative Commons Attribution License (http:// creativecommons.org/licenses/by/4.0/) which permits unrestricted use, distribution, and production in any medium, provided the author of the original work and original publication source are properly credited. 
for the batch arrival $G e o^{X} / G e o / 1$ queue has been analyzed by Upadhyaya (2015) under EAS scheme.

In variant working vacation (VWV) policy, unlike the SWV or MWVs, a fixed number of consecutive vacations, say $K$, are taken by the server if the system remains empty at the end of previous vacation termination epoch. This kind of vacation schedule is investigated by Zhang and Tian (2001) for the $G e o / G / l$ queue with multiple adaptive vacations. Banik (2009) studied the infinite-buffer single server queue with variant of multiple vacation policy and batch Markovian arrival process by using matrix analytic method. For more literature on this work, see Ke and Chang (2009), Ke et al. (2010) and Wang et al. (2011). Zhang and Hou (2011) analyzed a steady-state GI/M/I/N queue with a variant multiple working vacation (VWV) by using matrix analytic method. Yue et al. (2014) analyzed the $\mathrm{M} / \mathrm{M} / 1$ queueing system with impatient customers and VV and obtained the closed-form expressions of the mean system sizes when the server is in different states using probability generating functions. A finite buffer $M / M / 1$ queue with VWV, balking and reneging has been analyzed by Vijaya Laxmi and Jyothsna (2014) obtaining the steady state probabilities using matrix form solutions.

The above literature indicates that discrete-time Geo/Geo/l queue with VWV has not been studied so far to the best of our knowledge. In digital communication systems, this type of service /vacation policy is important as it reduces the switching and operating cost in case of least loaded systems. Further, the study of VWV queue gives more room for flexible switching from vacation to regular busy period by adjusting the value of $K$ as desired by system design requirements.

The primary objectives of the present paper are:

1. to obtain the steady-state probabilities a discrete-time Geo/Geo/l queue with VWV under late arrival system with delayed access (LAS-DA) using probability generating functions; and

2. To study the VWV (K-Vacation) policy that covers both MWV $(\mathrm{K} \rightarrow \infty)$ and SWV $(\mathrm{K}=1)$.

3. to study performance characteristics and to develop a cost model to determine the optimum service rates that optimize the total expected cost using the quasi-Newton optimization method.

The present model finds applications in many real-life applications like (i) packet switching communication protocols; (ii) the repair garage for automobiles wherein a mechanic repairs the automobiles; (iii) manufacturing and transportation processes, etc.

\section{MODEL DESCRIPTION}

Let us consider a discrete-time Geo / Geo / 1 queueing system with VWVs under LAS-DA policy. The time is divided into constant length intervals (called slots) and the probability of an arrival and a departure occurring simultaneously is not zero. A potential arrival occurs in the interval $(t-, t)$ and potential departure occurs in $(t, t+)$. In order to formulate the model, the following assumptions have been made:

The arrival of a customer to the system occurs at the end of slot $t-$, where $t=0,1,2, \ldots$. Interarrival times $(A)$ of two successive arrivals are independent and identically distributed (i.i.d) random variables and follow geometric distribution with probability mass function (p.m.f) (Figure 1).

$$
P\{A=m\}=\lambda \bar{\lambda}^{m-1}, m \geq 1,0<\lambda<1 .
$$

where for any real number $x \in[0,1]$, we denote $\bar{x}=1-x$. 
In variant working vacation policy, the server is allowed to take at most $K$ consecutive $W V s$, if the system remains empty at the end of a vacation. Suppose the beginning and ending of vacation occur at the epoch which is similar to $t$ in shape. The service is provided with rate $\mu$ and at the end of a service if there is no customer in the system, server begins working vacation, i.e., he remains dormant between the service completion epoch in $(t, t+)$ and the next arrival epoch in $((t+1)-, t+1)$. If some customers arrive in $((t+1)-, t+1)$, the dormant period will last until the beginning of epoch of service in $(t+1,(t+1)+)$; otherwise, the server takes a vacation at time $(t+1)$ immediately. During working vacation, if customer arrives, the server provides service with rate $\eta$. The distribution of service time during regular busy period $\left(S_{b}\right)$, service time during working vacation period $\left(S_{W V}\right)$ and vacation times $(V)$ are given, respectively by

$$
\begin{aligned}
& P\left\{S_{b}=m\right\}=\mu \bar{\mu}^{m-1}, 0<\mu<1 ; P\left\{S_{W V}=m\right\}=\eta \bar{\eta}^{m-1}, 0<\eta<1 \\
& P\{V=m\}=\phi \bar{\phi}^{m-1}, 0<\phi<1, m \geq 1 .
\end{aligned}
$$

If the server instantly finds a customer at working vacation completion, it returns to regular busy period; otherwise, takes working vacations sequentially until a maximum of $K$ vacations completion, after which the server comes to regular busy period and stays idle till a customer arrives. Variant working vacation generates multiple working vacation when $K \rightarrow \infty$ and single working vacation when $K$ is equal to 1 . Further, all the stochastic processes are assumed to be mutually independent. In addition, the service discipline is first in first out (FIFO).

\section{ANALYSIS OF THE MODEL}

In this section, we develop the difference equations for the probability generating functions (PGF) of the steady-state probabilities. Further, we procure the mean system size when the server is in different states.

Figure 1. Various time epochs in LAS - DA

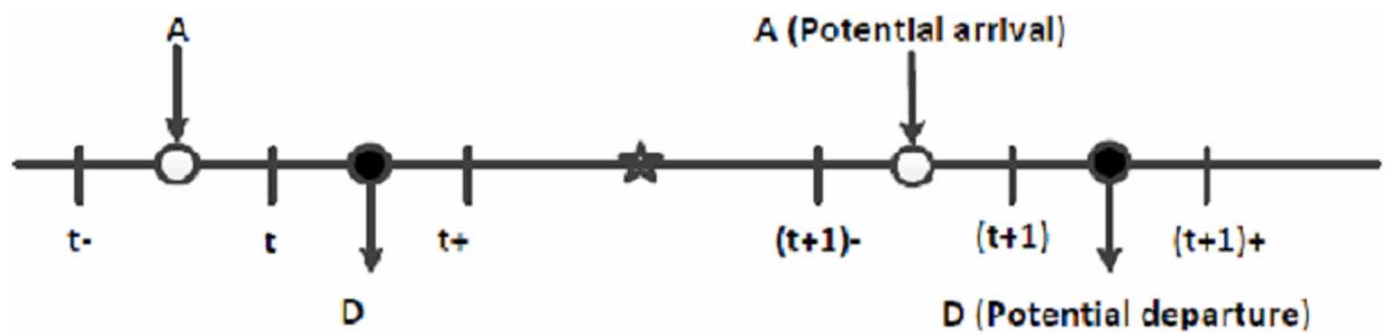

$$
\begin{array}{cl}
0 & \text { : Potential arrival epoch } \\
& \text { : Potential departure epoch } \\
(t+,(t+1)-) & : \text { Outside observer's interval } \\
t+\quad: \text { Epoch after a potential departure } \\
\text { t- } \quad \text { : Epoch prior to a potential arrival }
\end{array}
$$




\section{Balance Equations}

Let $L_{t}$ denote the number of customers in the system at time $t+$ and $J_{t}$ denote the status of the server at time $t+$, which is defined as follows:

$J_{n}= \begin{cases}j, & \text { the server is on }(j+1) \text { th } W V \text { at time } t+\text { for } j=0,1, \cdots K-1 \\ K, & \text { the server is idle or busy at time } t+\end{cases}$

The process $\left(\left(L_{n}, J_{n}\right), n \geq 0\right)$ defines a discrete-time Markov process with state space

$$
\text { (c) }=\{(n, j) ; n \geq 0, j=0,1, \cdots K\} \text {. }
$$

Let $P_{n, j}=\operatorname{Prob}\left\{L_{n}=n, J_{n}=j\right\} ; n \geq 0, j=0,1, \cdots K$, denote the steady-state probabilities of the process $\left\{\left(L_{n}, J_{n}\right), n \geq 0\right\}$. The set of balance equations can be written as:

$$
\begin{aligned}
& P_{0,0}=\bar{\phi} \bar{\lambda}\left(P_{0,0}+\eta P_{1,0}\right)+\bar{\lambda} \mu P_{1, K} \\
& P_{1,0}=\bar{\phi} \lambda P_{0,0}+\bar{\phi}(\bar{\lambda} \bar{\eta}+\lambda \eta) P_{1,0}+\bar{\phi} \bar{\lambda} \eta P_{2,0} \\
& P_{n, 0}=\bar{\phi} \lambda \bar{\eta} P_{n-1,0}+\bar{\phi}(\bar{\lambda} \bar{\eta}+\lambda \eta) P_{n, 0}+\bar{\phi} \bar{\lambda} \eta P_{n+1,0}, n \geq 2
\end{aligned}
$$

$$
P_{0, j}=\bar{\phi} \bar{\lambda}\left(P_{0, j}+\eta P_{1, j}\right)+\phi \bar{\lambda}\left(P_{0, j-1}+\eta P_{1, j-1}\right), 1 \leq j \leq K-1
$$

$$
P_{1, j}=\bar{\phi} \lambda P_{0, j}+\bar{\phi}(\bar{\lambda} \bar{\eta}+\lambda \eta) P_{1, j}+\bar{\phi} \bar{\lambda} \eta P_{2, j}, 1 \leq j \leq K-1
$$

$$
P_{n, j}=\bar{\phi} \lambda \bar{\eta} P_{n-1, j}+\bar{\phi}(\bar{\lambda} \bar{\eta}+\lambda \eta) P_{n, j}+\bar{\phi} \bar{\lambda} \eta P_{n+1, j}, 1 \leq j \leq K-1
$$

$$
P_{0, K}=\bar{\lambda} P_{0, K}+\phi \bar{\lambda}\left(P_{0, K-1}+\eta P_{1, K-1}\right)
$$

$$
P_{1, K}=\lambda P_{1, K}+\bar{\lambda} \mu P_{2, K}+(\bar{\lambda} \bar{\mu}+\lambda \mu) P_{1, K}+\phi \sum_{j=0}^{K-1}\left[\lambda P_{0, j}+(\bar{\lambda} \bar{\eta}+\lambda \eta) P_{1, j}+\bar{\lambda} \eta P_{2, j}\right]
$$




$$
P_{n, K}=\lambda \bar{\mu} P_{n-1, K}+\bar{\lambda} \mu P_{n+1, K}+(\bar{\lambda} \bar{\mu}+\lambda \mu) P_{n, K}+\phi \sum_{j=0}^{K-1}\left[\lambda \bar{\eta} P_{n-1, j}+(\bar{\lambda} \bar{\eta}+\lambda \eta) P_{n, j}+\bar{\lambda} \eta P_{n+1, j}\right], n \geq 2
$$

and the normalization condition

$\sum_{j=0}^{K} \sum_{n=0}^{\infty} P_{n, j}=1$

The steady-state probabilities are obtained by solving the above system of Equations (1) to (9) using $P G F$. Let us define $P G F$ of $P_{n, j}$ as

$G_{j}(z)=\sum_{n=0}^{\infty} P_{n, j} z^{n},|z| \leq 1, j=0,1, \cdots K-1$

By using the probability generating function on the balance Equations (1) to (9), we obtain the following results:

The detailed computation of steady-state probabilities, the mean system sizes and other interesting results are presented in Appendix A.

\section{Performance Measures and Cost Model}

In this section, some performance measures and their monotonicity with respect to $K$ are presented. The probability that the server is in WV state is denoted by $P_{W V}$ and the probability that the server is in regular busy period is denoted by $P_{B u s y}$ such that $P_{W V}+P_{\text {Busy }}=1$. Again, the probability that the system is empty and the server is in $\mathrm{WV}$ is denoted by $P_{e}$. The probability that the system is empty during regular busy period (in which case we say the server is idle) is $P_{0, K}$. The probability that the server is busy serving a customer during regular busy period is denoted by $P_{b}$ where $P_{\text {Busy }}=P_{b}+P_{0, K}$. The average number of customers in the system and that in the queue are, respectively denoted by $E[L]$ and $E\left[L_{q}\right]$. A cost model is also developed to determine the optimal service rate during WV.

\section{Performance Measures}

- The Probability that the Server is Idle: The probability that the server is idle during regular busy period is given from Equation (48) as

We observe that $\left(1-B^{K}\right)$ decreases with $K$. Assuming $K$ as a continuous variable we have $\frac{d P_{0, K}}{d K}<0$ which shows that $P_{0, K}$ is a decreasing function of $K$.

- Probability when the Server is on Working Vacation: The probability of the server being in WV $\left(\mathrm{P}_{\mathrm{WV}}\right)$ is given from Equation (50) as 


$$
P_{W V}=\sum_{j=0}^{K-1} G_{j}(1)=\frac{\left(\phi+\lambda\left(1-z^{*}\right) \bar{\phi}\right)(\mu-\lambda)\left(1-B^{K}\right)}{(\mu-\eta) \lambda\left(1-z^{*}\right) \bar{\phi}\left(1-B^{K}\right)+\phi \mu\left(1-B^{K} z^{*}\right)}
$$

We see that $P_{W V}$ increases with $K$ because of the decrease of $P_{0, K}$ with respect to $K$.

- Probability of Busy Server during Regular Busy Period: The probability of busy server is denoted by $P_{b}$ and given by

$$
P_{b}=1-P_{0, K}-P_{W V}=\frac{(\lambda-\eta) \lambda\left(1-z^{*}\right) \bar{\phi}\left(1-B^{K}\right)+\phi \lambda\left(1-B^{K} z^{*}\right)}{(\mu-\eta) \lambda\left(1-z^{*}\right) \bar{\phi}\left(1-B^{K}\right)+\phi \mu\left(1-B^{K} z^{*}\right)}
$$

From the fact that $P_{0, K}$ decreases, $P_{W V}$ increases and $\frac{d P_{b}}{d K}<0$ with $K$, so $P_{b}$ decreases with $K$.

- Probability when the Server is in Regular Busy Period: The probability that the server is in regular busy period is given by

$$
P_{\text {Busy }}=\sum_{n=0}^{\infty} P_{n, K}=P_{0, K}+P_{b}, \text { where } P_{\text {Busy }}+P_{W V}=1 \text {. }
$$

- Proportion of Customers Served: Clearly, the expected number of customers served per unit of time is $\mu P_{b}$, implying that the proportion of customers served is given by

$$
P_{s}=\frac{\mu P_{b}}{\lambda}=\frac{P_{b}}{\rho}
$$

where $\rho=\lambda / \mu$ and $P_{b}$ is given by Equation (12).

- The Probability when the System is Empty and the Server is on Working Vacation: The probability when the system is empty and the server is on working vacation is given by

$$
P_{e}=\sum_{j=0}^{K-1} \frac{\phi(\mu-\lambda)\left(1-B^{K}\right)}{(\mu-\eta) \lambda\left(1-z^{*}\right) \bar{\phi}\left(1-B^{K}\right)+\phi \mu\left(1-B^{K} z^{*}\right)}
$$




$$
P_{e}=\frac{B}{\left(\bar{\lambda}+\lambda z^{*}\right)} P_{W V},
$$

where $P_{W V}$ is given in Equation (11).

- The Average Number of Customers in the System: The average number of customers in the system $(E[L])$ is given by

$$
E[L]=E\left[L_{W V}\right]+E\left[L_{K}\right]
$$

where $E\left[L_{W V}\right]$ (the mean system size when the server is in WV) and $E\left[L_{K}\right]$ (the mean system size when the server is in regular busy period) are obtained from Equations (51) and (54) in appendix A.

- The Average Number of Customers in the Queue $\left(E\left[L_{q}\right]\right)$ :

$$
E\left[L_{q}\right]=\sum_{j=0}^{K} \sum_{n=1}^{\infty}(n-1) P_{n, j}
$$

\section{Cost Model}

In this subsection, an expected cost function is formulated, in which mean service rate $(\zeta)$ during working vacation is the control variable. Assume that

$C_{1} \equiv$ service cost per unit time when the server is busy,

$C_{2} \equiv$ service cost per unit time when the server is on working vacation,

$C_{3}$ 三 fixed cost per unit time when the server is on working vacation,

$C_{4} \equiv$ cost per unit time when the server is idle,

$C_{5} \equiv$ cost per unit time when the server is idle during working vacation, and

$C_{6} \equiv$ cost per unit time of every customer in the queue and waiting for service.

Using the definition of each cost element and its corresponding system performance measures, the total expected cost function per unit time is given by

$$
f(\eta)=C_{1} \mu P_{b}+\left(C_{2} \eta+C_{3} \phi\right) P_{W V}+C_{4} P_{0, K}+C_{5} P_{e}+C_{6} E\left[L_{q}\right] .
$$

Our objective is to determine the optimal mean service rate $\eta^{*}$ during working vacation that minimizes the cost function $f[\eta]$. Quasi-Newton method is employed to solve the above optimization problem, as the analytical computation of derivatives of the above expected cost function is a difficult task. 


\section{Quasi-Newton Method}

If the function being minimized $f(\eta)$ is not available in closed form or is difficult to differentiate, the derivatives $f^{\prime}(\eta)$ and $f^{\prime \prime}(\eta)$ of the Equation (18) can be approximated by the finite difference formula

$$
f^{\prime}\left(\eta_{i}\right)=\frac{f\left(\eta_{i}+" \eta\right)-f\left(\eta_{i}-" \eta\right)}{2 " \eta} ; f^{\prime \prime}\left(\eta_{i}\right)=\frac{f\left(\eta_{i}+" \eta\right)-2 f\left(\eta_{i}\right)+f\left(\eta_{i}-" \eta\right)}{(" \eta)^{2}}
$$

where " $\zeta$ is a small step size. The iterative process of quasi-Newton method is given by

$$
\eta_{i+1}=\eta_{i}-\frac{f^{\prime}\left(\eta_{i}\right)}{f^{\prime \prime}\left(\eta_{i}\right)}, i=1,2, \cdots
$$

The iterative process stops when either $\left|\eta_{i+1}-\eta_{i}\right|<\epsilon$ or after performing a pre-specified number of iteration. Here $\epsilon$ is the error tolerance, see details on Quasi-Newton method in Rao (2009)

\section{NUMERICAL RESULTS}

In this section, our aim is to study the parameter impact on the system performance through numerical simulations. Numerical illustrations are provided by considering following parameters: $C_{1}=6.5, C_{2}=2.5, C_{3}=3.5, C_{4}=1.5, C_{5}=1.0$ and $C_{6}=3.0$ and the other parameters as $\lambda=0.2, \mu=0.55, K=6, \phi=0.3$ and $\eta=0.2$. These numerical values for the system parameters and the cost coefficients are all taken randomly for demonstration purpose without violating the assumptions considered and the stability condition $\rho=\frac{\lambda}{\mu}<1$.

The effect of service rate during working vacation $\eta$ on the total expected cost function $(f(\eta))$ is shown in Figure 2. The convexity of the curve shows that there exists a value of $\eta$ that minimizes the total expected cost function for the chosen set of model parameters. Quasi-Newton method is adopted to find the optimal value by choosing the initial point $\eta=0.22$, step size " $\eta=0.0001$ and the stopping tolerance $\epsilon=10^{-5}$. After three iterations, the minimum expected operating cost per unit time converges to the solution $f(\eta)=3.28189$ for $\eta^{*}=0.230138$ as shown in Figure 2 .

Figure 3 illustrates the effect of service rate during working vacation $\eta$ on the mean system size $(E[L])$ for various vacation parameters $\phi$. (i) Obviously, the declining trend indicates that system size reduces with faster services. (ii) With regard to $\phi$, we see that $E[L]$ decreases as $\phi$ increases as long as $\mu \geq \eta$. This trend is completely reversed when $\mu<\eta$. The increase in vacation rate makes frequent switch over of the server to regular busy period. Thus, if $\mu<\eta$, the system tends to be slower and so $E[L]$ increases; whereas, if $\mu>\eta$, the system becomes faster more often. Thus, $E[L]$ decreases. Also the intersection point at $\eta=\mu=0.55$ is an equilibrium point where the mean system size remains unchanged regardless of the vacation rates.

Figure 4 depicts the impact of $P_{b}$ and $P_{W V}$ with respect to $\lambda$ for different $\phi$. We observe that $P_{b}$ increases with $\lambda$, whereas $P_{W V}$ decreases. Obviously, a larger arrival rate implies the system in 


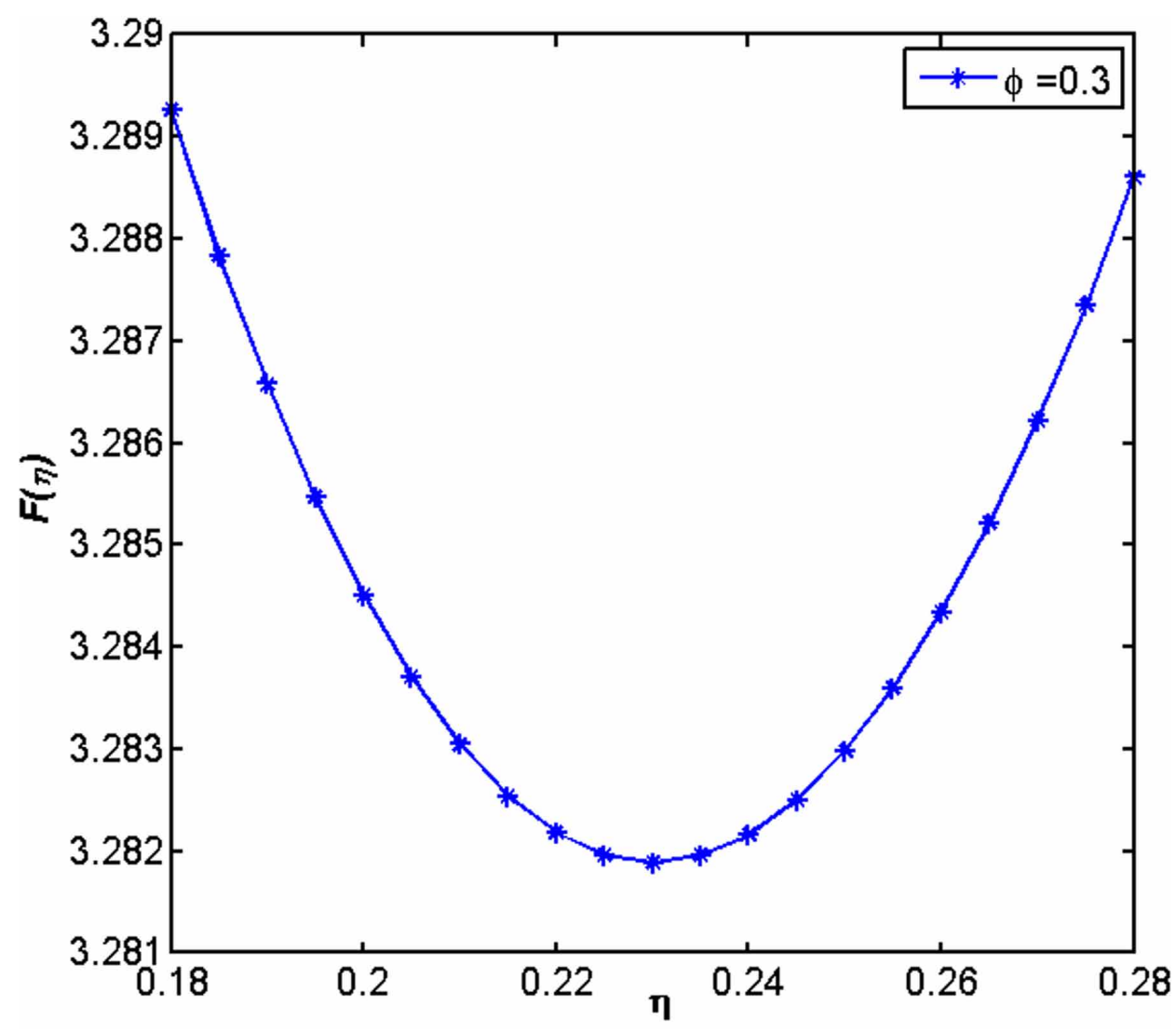

the busy state until the server finds empty system, which is reflected in the increase in $P_{b}$ and a decrease in $P_{W V}$.

For a fixed $\lambda, P_{b}$ increases and $P_{W V}$ decreases with the increase of $\phi$. On the other hand, for a given $\phi$, the curves corresponding to $P_{b}$ and $P_{W V}$ intersect at some value of $\lambda$ in such a way that this value of $\lambda$ decreases as $\phi$ increases. This is justifiable since increasing $\phi$ increases $P_{b}$ whereas decreasing $\lambda$ increases $P_{W V}$. Thus, a point of equilibrium is reached at the point of intersection.

The effect of $\lambda$ and $\mu$ on $E[L]$ is depicted in Figure 5. One may observe that $E[L]$ increases as $\lambda$ increases whereas it decreases with $\mu$. Further, the intersection point $(\lambda, \mu)=(0.37,0.57)$ gives the optimum value of $E[L]$. The impact of service rate $\mu$ on $E\left[L_{q}\right]$ is shown in Figure 6 for $K=1$ (single WV) and $K=7(V W V)$. We observe that as $\mu$ increases, $E\left[L_{q}\right]$ decreases for a fixed $K$ and for a particular $\mu, E\left[L_{q}\right]$ increases as $K$ increases. This is because, the server returns to regular busy period only if it finds at least one customer at the completion of a working vacation. Otherwise, he takes next WV until the maximum number of vacations $K$ is reached. Therefore, server stays longer in WV for larger values of $K$. As a result, the expected queue size increases with the increase in $K$, as shown in Figure 6. 
Figure 3. Effect of $\operatorname{\eta on} E[L[$

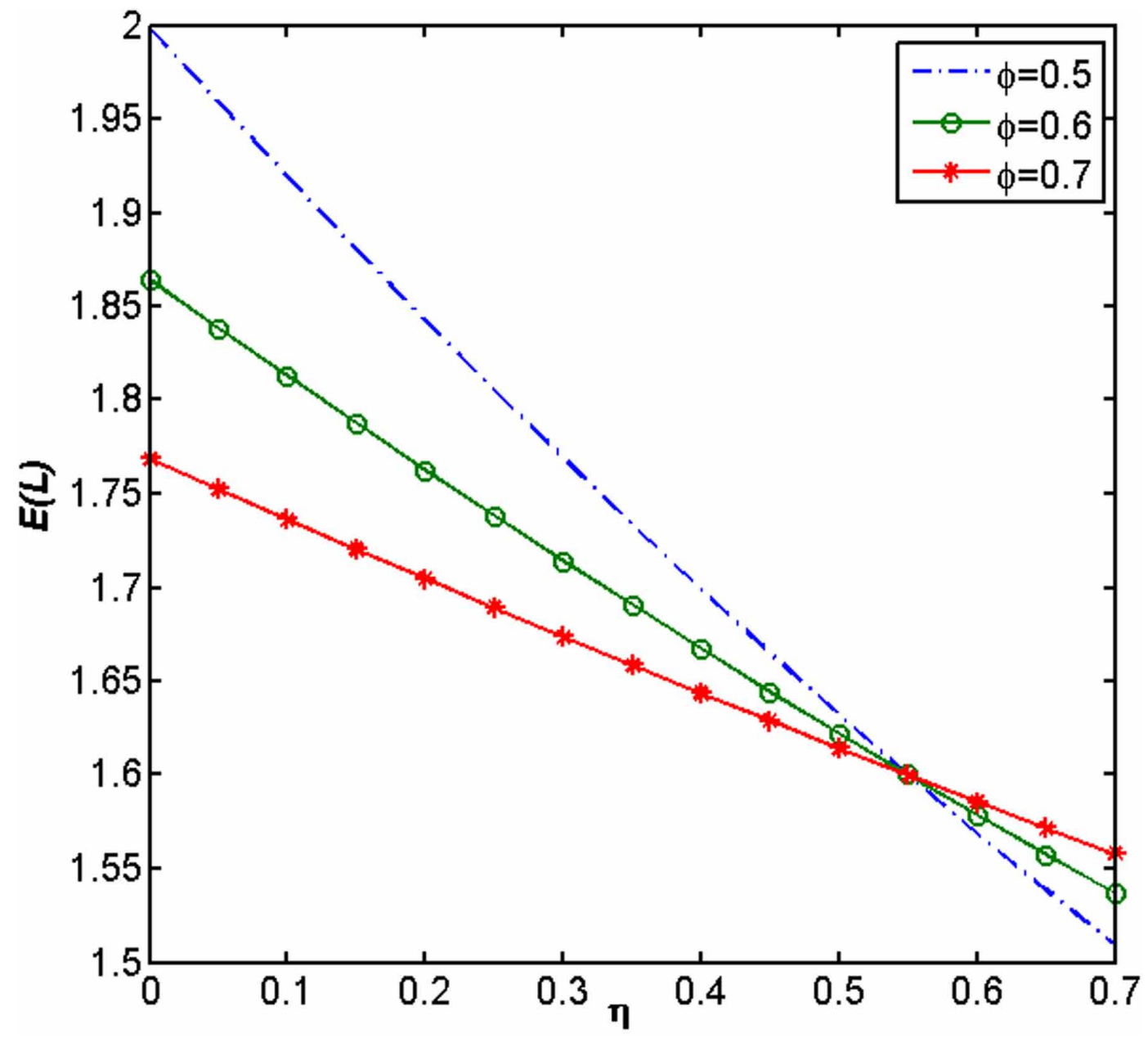

\section{CONCLUSION}

In this paper, a $G e o / G e o / 1$ queueing system with variant working vacations is studied. PGFs of the number of customers in the system and the corresponding mean system sizes when the server is in different states have been derived. The closed-form expressions for some other performance measures are derived and the cost optimization has been done using the simple but effective quasi-Newton method, and the optimum values of the service rates during working vacation have been obtained. The technique adopted in this paper can be applied to analyze models like $G e o^{X} / G / 1$ queue with variant working vacations, impatient customer $G I^{x} / G e o / 1$ queue with variant working vacations. Also, the present model can be generalized to a renewal input impatient customer queue with Bernoulli schedule vacation interruption.

\section{ACKNOWLEDGMENT}

The authors are thankful to the Editor and the anonymous referees for their valuable comments and suggestions which have helped in improving the quality and the presentation of the paper. 
Figure 4. Effect of $\lambda o n P_{b}$ and $P_{W V}$

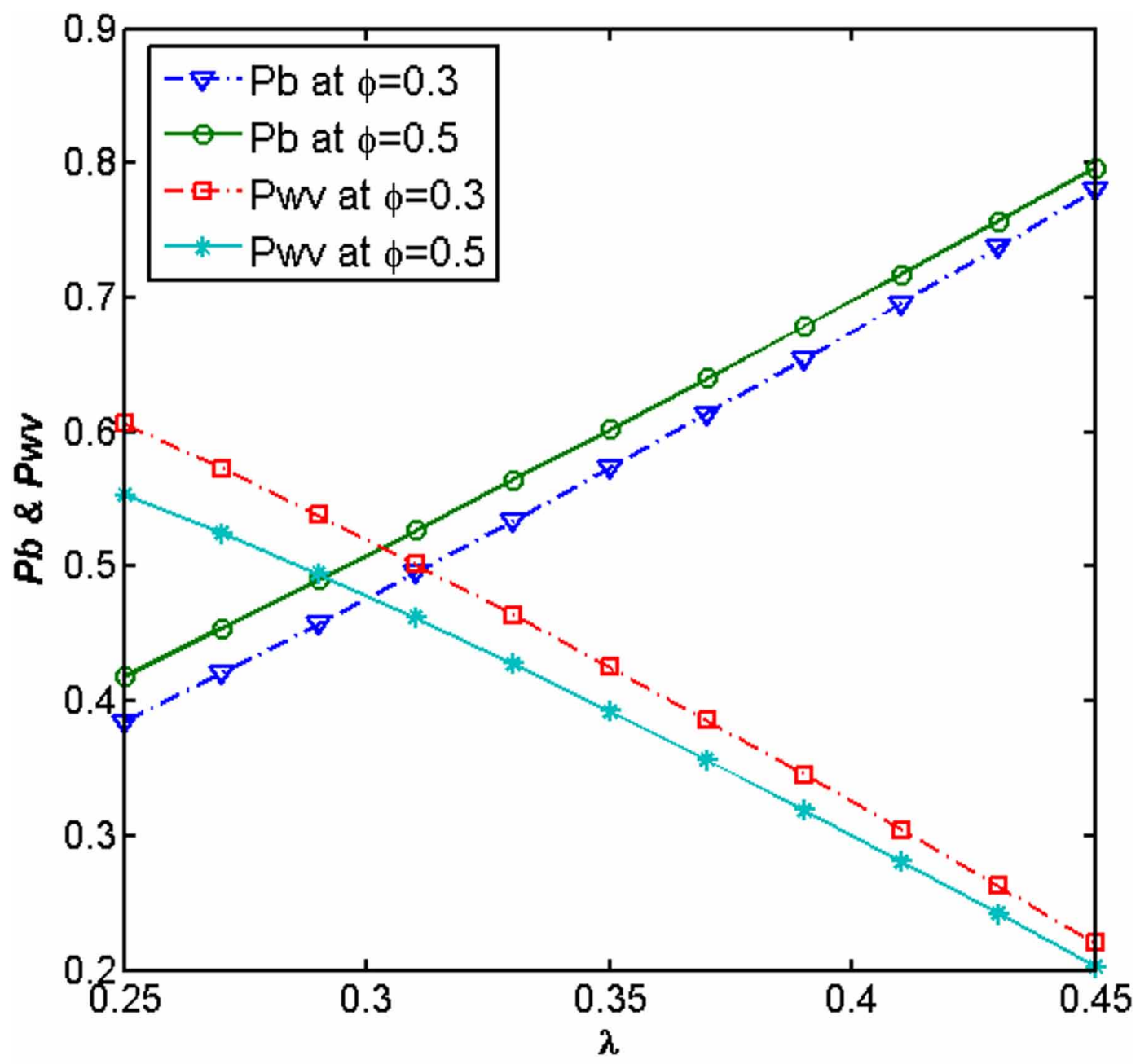


Figure 5. Effect of $\lambda$ and $\mu$ on $E[L]$

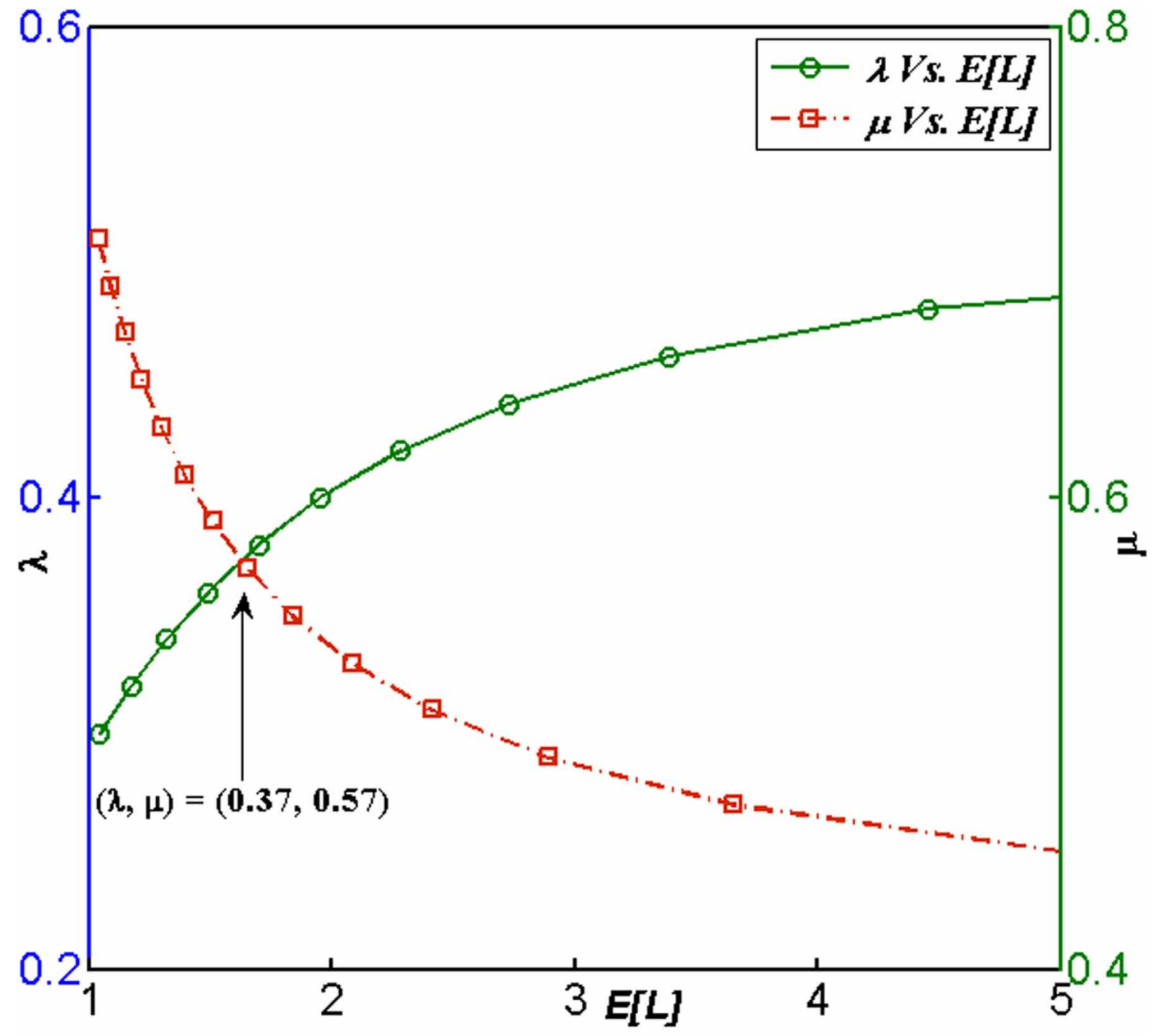


Figure 6. Effect of $\mu$ on $E\left[L_{q}\right]$

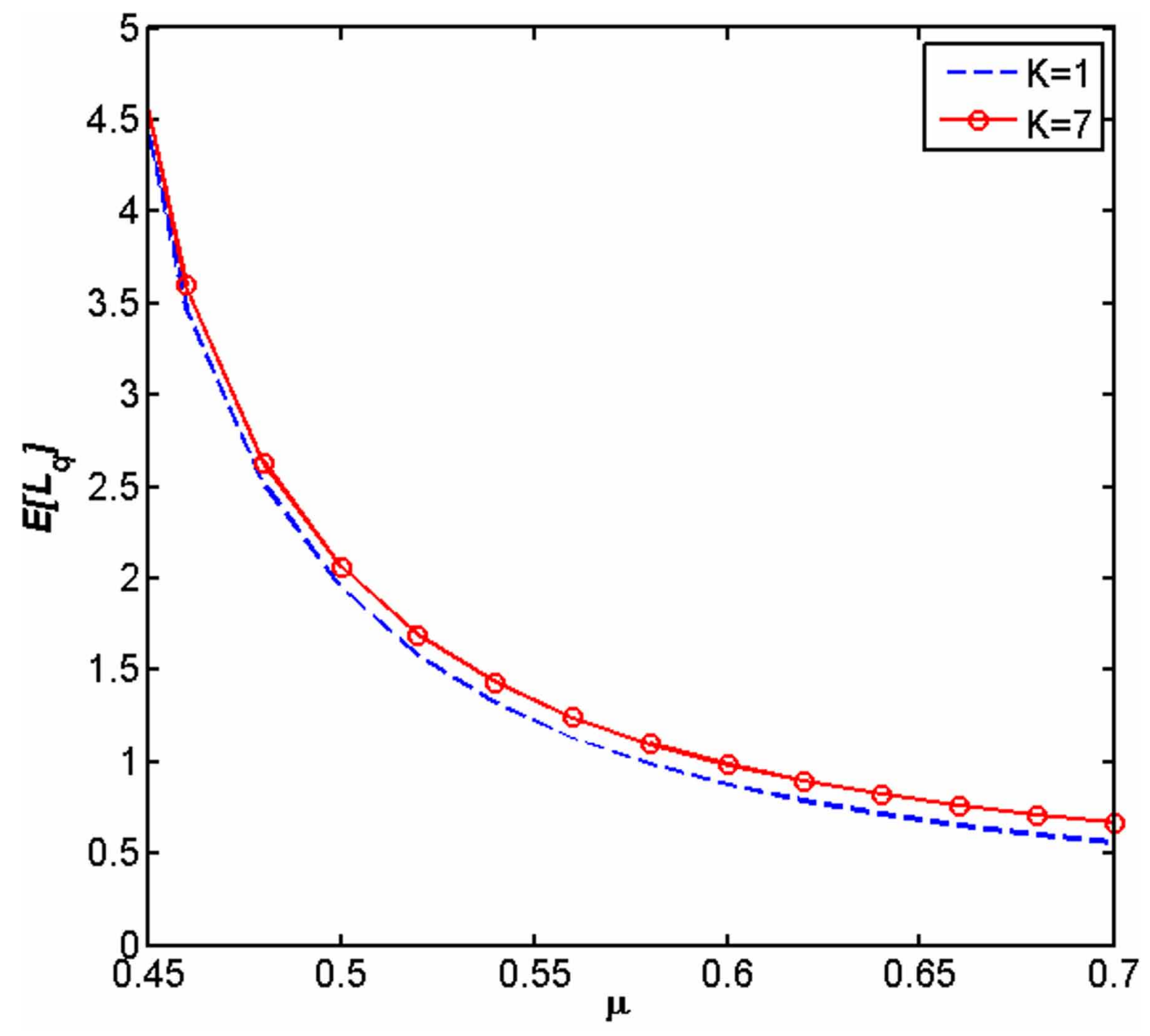




\section{REFERENCES}

Alfa, A. S. (2003). Vacation models in discrete time. Queueing Systems, 44(1), 5-30. doi:10.1023/A:1024028722553

Banik, A. D. (2009). The infinite-buffer single server queue with a variant of multiple vacation policy and batch Markovian arrival process. Applied Mathematical Modelling, 33(7), 3025-3039. doi:10.1016/j.apm.2008.10.021

Banik, A. D., Gupta, U. C., \& Pathak, S. S. (2007). On the GI/M/1/N queue with multiple working vacationsanalytic analysis and computation. Applied Mathematical Modelling, 31(9), 1701-1710. doi:10.1016/j. apm.2006.05.010

Doshi, B. T. (1986). Queueing systems with vacations-A survey. Queueing Systems, 1(1), 29-66. doi:10.1007/ BF01149327

Hunter, J. (1983). Mathematical Techniques of Applied Probability: Vol. 2. Discrete Time Models: Techniques and Applications. New York: Academic Press.

Ke, J. C., Huang, K. B., \& Pearn, W. L. (2010). Randomized policy of a Poisson input queue with J vacations. Journal of Systems Science and Systems Engineering, 19(1), 50-71. doi:10.1007/s11518-010-5124-z

Li, J., \& Tian, N. (2008). Analysis of the discrete-time Geo/Geo/1 queue with single working vacation. Quality Technology \& Quantitative Management, 5(1), 77-89. doi:10.1080/16843703.2008.11673177

Li, J. H., Tian, N., Zhang, Z. G., \& Luh, H. P. (2009). Analysis of the $M / G / 1$ queue with exponentially working vacations - A matrix analytic approach. Queueing Systems, 61(2-3), 139-166. doi:10.1007/s11134-008-9103-8

Li, J. H., \& Tian, N. S. (2007). The discrete-time GI/Geo/1 queue with working vacations and vacation interruption. Applied Mathematics and Computation, 185(1), 1-10. doi:10.1016/j.amc.2006.07.008

Li, J. H., Tian, N. S., \& Liu, W. Y. (2007). Discrete-time GI/Geo/1 queue with multiple working vacations. Queueing Systems, 56(1), 53-63. doi:10.1007/s11134-007-9030-0

Liu, W., Xu, X., \& Tian, N. (2007). Stochastic decompositions in the $M / M / 1$ queue with working vacations. Operations Research Letters, 35(5), 595-600. doi:10.1016/j.orl.2006.12.007

Meisling, T. (1958). Discrete-time queueing theory. Operations Research, 6(1), 96-105. doi:10.1287/opre.6.1.96

Rao, S. S. (2009). Engineering Optimization: Theory and Practice. New Jersey: Wiley. doi:10.1002/9780470549124

Servi, L. D., \& Finn, S. G. (2002). M/M/1 queues with working vacations (M/M/1/WV). Performance Evaluation, 50(1), 41-52. doi:10.1016/S0166-5316(02)00057-3

Takagi, H. (1993). Queueing Analysis: Discrete Time Systems (Vol. 3). Amsterdam: Elsevier Science Publishers.

Tian, N., Ma, Z., \& Liu, M. (2008). The discrete time Geom/Geom/1 queue with multiple working vacations. Applied Mathematical Modelling, 32(12), 2941-2953. doi:10.1016/j.apm.2007.10.005

Tian, N., \& Zhang, Z. G. (2002). The discrete-time GI/Geo/l queue with multiple vacations. Queueing Systems, 40(3), 283-294. doi:10.1023/A:1014711529740

Upadhyaya, S. (2015). Working vacation policy for a discrete-time $G e o^{x} / G e o / 1$ retrial queue. OPSEARCH, 52(4), 650-669. doi:10.1007/s12597-015-0200-2

Vijaya Laxmi, P., \& Jyothsna, K. (2014). Performance analysis of variant working vacation queue with balking and reneging. International Journal of Mathematics in Operational Research, 6(4), 505-521. doi:10.1504/ IJMOR.2014.063158

Vijaya Laxmi, P., \& Jyothsna, K. (2014). Performance analysis of variant working vacation queue with balking and reneging. International Journal of Mathematics in Operational Research, 6(4), 505-521. doi:10.1504/ IJMOR.2014.063158

Vijaya Laxmi, P., Jyothsna, K., \& Seleshi, D. (2015). Analysis of a discrete-time working vacation queue with balking. OPSEARCH, 52(3), 562-581. doi:10.1007/s12597-014-0191-4

Wang, T. Y., Ke, J. C., \& Chang, F. M. (2011). On the discrete-time Geo/G/1 queue with randomized vacations and at most J vacations. Applied Mathematical Modelling, 35(5), 2297-2308. doi:10.1016/j.apm.2010.11.021 
Wu, D., \& Takagi, H. (2006). M/G/1 queue with multiple working vacations. Performance Evaluation, 63(7), 654-681. doi:10.1016/j.peva.2005.05.005

Yue, D., Yue, W., Saffer, Z., \& Chen, X. (2014). Analysis of an M/M/1 queueing system with impatient customers and a variant of multiple vacation policy. Journal of Industrial and Management Optimization, 10(1), 89-112. doi:10.3934/jimo.2014.10.89

Zhang, M., \& Hou, Z. (2011). Steady state analysis of the GI/M/1/N queue with a variant of multiple working vacations. Computers \& Industrial Engineering, 61(4), 1296-1301. doi:10.1016/j.cie.2011.08.002

Zhang, Z. G., \& Tian, N. (2001). Discrete time Geo/G/1 queue with multiple adaptive vacations. Queueing Systems, 38(4), 419-429. doi:10.1023/A:1010947911863 


\section{APPENDIX A. DETAILS OF THE PROBABILITY GENERATING FUCTION METHOD}

In the appendix below, we present the details of the probability generating function method to obtain the steady-state results and the aggregate probabilities during regular busy and WVs.

Multiplying Equations (1), (2) and (3) of the balance equations by $z^{n}$, and summing over all possible values of $n$ and re-arranging the terms, we get

$$
\left[\bar{\phi} F_{\eta}(z)-z\right] G_{0}(z)=\bar{\phi}\left(F_{\eta}(z)-(\bar{\lambda}+\lambda z) z\right) P_{0,0}-\bar{\lambda} \mu z P_{1, K}
$$

Similarly, from the balance Equations (4), (5) and (6) and (7), (8) and (9), respectively, we get

$$
\left[\bar{\phi} F_{\eta}(z)-z\right] G_{j}(z)=\bar{\phi}\left(F_{\eta}(z)-(\bar{\lambda}+\lambda z) z\right) P_{0, j}-\phi \bar{\lambda} z\left(P_{0, j-1}+\eta P_{1, j-1}\right), 1 \leq j \leq K-1
$$

$$
\begin{aligned}
& {\left[F_{\mu}(z)-z\right] G_{K}(z)+\phi F_{\eta}(z) \sum_{j=0}^{K-1} G_{j}(z)=\left(F_{\mu}(z)-(\bar{\lambda}+\lambda z) z\right) P_{0, K}+\phi\left(F_{\eta}(z)-\lambda z^{2}\right) \sum_{j=0}^{K-2} P_{0, j}} \\
& +\phi\left(F_{\eta}(z)-\left(F_{\mu}(z)-(\bar{\lambda}+\lambda z) z\right) P_{0, K-1}+\left[\bar{\lambda} \mu P_{1, K}+\phi \bar{\lambda} \eta \sum_{j=0}^{K-2} P_{1, j}\right] z\right.
\end{aligned}
$$

w h e r e $\quad F_{\zeta}(z)=\lambda \bar{\eta} z^{2}+(\bar{\lambda} \bar{\eta}+\lambda \eta) z+\bar{\lambda} \eta, \quad F_{\mu}(z)=\lambda \bar{\mu} z^{2}+(\bar{\lambda} \bar{\mu}+\lambda \mu) z+\bar{\lambda} \mu \quad$ a n d $F_{\eta}(1)=F_{\mu}(1)=1$. By taking $\mathrm{z}=1$ into Equations (19) and (20), we obtain

$\phi G_{0}(1)=\bar{\lambda} \mu P_{1, K}$

$G_{j}(1)=\bar{\lambda}\left(P_{0, j-1}+\eta P_{1, j-1}\right), j=1,2, \cdots K-1$.

By using Equations (22) and (23), we obtain

$$
\bar{\lambda} \mu P_{1, K}+\phi \bar{\lambda} \sum_{j=1}^{K-1}\left(P_{0, j-1}+\eta P_{1, j-1}\right)=\phi \sum_{j=0}^{K-1} G_{j}(1) .
$$

Substituting above Equation in (21), we get

$$
G_{K}(z)=\frac{\left.\left(F_{\mu}(z)-\bar{\lambda}+\lambda z\right) z\right) P_{0, K}-\phi\left[F_{\eta}(z) \sum_{j=0}^{K-1} G_{j}(1)\right]+N_{1}}{F_{\mu}(z)-z}
$$


where $N_{1}=\phi\left(F_{\eta}(z)-(\bar{\lambda}+\lambda z) z\right) \sum_{j=0}^{K-1} P_{0, j}$. Setting $\mathrm{z}=1$ and applying L'Hospital rule, we get

$G_{K}(1)=\frac{\phi \sum_{j=0}^{K-1} G_{J}^{\prime}(1)+\phi\left[(\lambda-\eta) \sum_{j=0}^{K-1} G_{j}(1)+\eta \sum_{j=0}^{K-1} P_{0, j}\right]+\mu P_{0, K}}{(\mu-\lambda)}$

The average number of customers in the system during the jth WV denoted by $E\left[L_{j}\right]$ is given by $E\left[L_{j}\right]=G_{j}^{\prime}(1), j=0,1, \cdots K-1$. From Equation (19) and (22) we have for $z=1$

$G_{0}^{\prime}(1)=\frac{\bar{\phi}}{\phi}\left[(\lambda-\eta) G_{0}(1)+\zeta P_{0,0}\right]$

Similarly, from Equations (20) and (23), we get

$G_{j}^{\prime}(1)=\frac{\bar{\phi}}{\phi}\left[(\lambda-v) G_{j}(1)+\eta P_{0, j}\right], j=1,2, \cdots K-1$.

Equations (27) and (28) imply

$$
E\left[L_{j}\right]=G_{j}^{\prime}(1)=\frac{\bar{\phi}}{\phi}\left[(\lambda-\eta) G_{j}(1)+\eta P_{0, j}\right], j=1,2, \cdots K-1 .
$$

Therefore, the mean system size when the server is on working vacation, denoted by $E\left[L_{W V}\right]$, is obtained as

$$
E\left[L_{W V}\right]=\sum_{j=0}^{K-1} E\left[L_{j}\right]=\sum_{j=0}^{K-1} G_{j}^{\prime}(1)=\frac{\bar{\phi}}{\phi}\left[(\lambda-\eta) \sum_{j=0}^{K-1} G_{j}(1)+\eta \sum_{j=0}^{K-1} P_{0, j}\right]
$$

By using Equation (30), Equation (26) can be written as

$$
G_{K}(1)=\frac{(\lambda-\eta) \sum_{j=0}^{K-1} G_{j}(1)+\eta \sum_{j=0}^{K-1} P_{0, j}+\mu P_{0, K}}{\mu-\lambda}
$$

Now, we derive $\sum_{j=0}^{K-1} G_{j}(1)$ and $\sum_{j=0}^{K-1} P_{0, j}$. Adding Equations (19) and (20), we have

$$
\sum_{j=0}^{K-1} \frac{\bar{\phi}\left(F_{\eta}(z)-(\bar{\lambda}+\lambda z) z\right) \sum_{j=0}^{K-1} P_{0, j}-z\left[\bar{\lambda} \mu P_{1, K}+\phi \bar{\lambda} \sum_{j=1}^{K-1}\left(P_{0, j-1}+\eta P_{1, j-1}\right)\right]}{\left(\bar{\phi} F_{\eta}(z)-z\right)}
$$


Lemma 3.1 The equation $\bar{\phi} F_{\eta}(z)-z=0$ has only one real root $z=z^{*}$ in the interval $(0,1)$.

Proof: : Consider $f(z)=\bar{\phi} F_{\eta}(z)-z=\bar{\phi}\left(\lambda \bar{\eta} z^{2}+(\bar{\lambda} \bar{\eta}+\lambda \eta) z+\bar{\lambda} \eta\right)-z$

F o r $\quad$ a n y $\quad 0<z<1, \quad f^{\prime}(z)=\bar{\phi}(2 \lambda \bar{\eta} z+\bar{\lambda} \bar{\eta}+\lambda \eta)-1, \quad f^{\prime \prime}(z)=2 \bar{\phi} \lambda \bar{\eta}>0, \quad$ a n d $f(0)=\bar{\phi} \bar{\lambda} \eta>0, f(1)=-\phi<0$, indicating that the equation $f(z)=0$ has only one real root $z=z^{*}$ in the interval $(0,1)$.

As the denominator of (32) vanishes at $z=z^{*}$ and $G_{j}(z)$ are assumed to be analytic functions, the numerator should also be zero. Therefore,

$$
\bar{\lambda} \mu P_{1, K}+\phi \bar{\lambda} \sum_{j=0}^{K-1}\left(P_{0, j-1}+\eta P_{1, j-1}\right)=\left(1-\left(\bar{\lambda}+\lambda z^{*}\right) \bar{\phi}\right) \sum_{j=0}^{K-1} P_{0, j}
$$

Using Equation (33), Equation (32) can be written as

$$
\begin{aligned}
& \sum_{j=0}^{K-1} G_{j}(z)=\frac{\left[\bar{\phi}\left(F_{\eta}(z)-(\bar{\lambda}+\lambda z) z\right)-z\left(1-\left(\bar{\lambda}+\lambda z^{*}\right) \bar{\phi}\right)\right] \sum_{j=0}^{K-1} P_{0, j}}{\bar{\phi} F_{\eta}(z)-z} \\
& \sum_{j=0}^{K-1} G_{j}(1)=\frac{\phi+\lambda\left(1-z^{*}\right) \bar{\phi}}{\phi} \sum_{J=0}^{K-1} P_{0, j}
\end{aligned}
$$

Using Equations (34), (31) and the normalization condition $\sum_{j=0}^{K-1} G_{j}(1)+G_{K}(1)=1$, we have

$$
\sum_{j=0}^{K-1} P_{0, j}=\frac{\phi\left(\mu-\lambda-\mu P_{0, K}\right)}{(\mu-\eta) \lambda\left(1-z^{*}\right) \bar{\phi}+\mu \phi}
$$

Substituting Equation (35) in (34) and (30) yields

$$
\sum_{j=0}^{K-1} G_{j}(1)=\frac{\phi+\lambda\left(1-z^{*}\right) \bar{\phi}\left(\mu-\lambda-\mu P_{0, K}\right)}{(\mu-\eta) \lambda\left(1-z^{*}\right) \bar{\phi}+\mu \phi}
$$

and

$$
E\left[L_{W V}\right]=\frac{\bar{\phi}}{\phi} \frac{\left((\lambda-\eta) \lambda\left(1-z^{*}\right) \bar{\phi}+\phi \lambda\right)\left(\mu-\lambda-\mu P_{0 K}\right)}{(\mu-\eta) \lambda\left(1-z^{*}\right) \bar{\phi}+\mu \phi}
$$

From Lemma $1, z=z^{*}$ is the root of $\bar{\phi} F_{\eta}(z)-z=0$, which gives 


$$
z^{*}=\frac{(1-\bar{\phi}(\bar{\lambda} \bar{\eta}+\lambda \eta))-\sqrt{(1-\bar{\phi}(\bar{\lambda} \bar{\eta}+\lambda \eta))^{2}-4\left(\bar{\phi}^{2} \lambda \bar{\lambda} \bar{\eta}\right)}}{2 \bar{\phi} \lambda \bar{\eta}}<1
$$

Taking $z=z^{*}$ in Equations (19) and (20), we get

$$
\begin{aligned}
P_{0,0} & =\frac{\bar{\lambda} \mu P_{1, K}}{\phi+\lambda\left(1-z^{*}\right) \bar{\phi}}, \\
P_{0, j} & =\frac{\phi \bar{\lambda}\left(P_{0, j-1}+\eta P_{1, j-1}\right)}{\phi+\lambda\left(1-z^{*}\right) \bar{\phi}}, j=1,2, \cdots K-1 .
\end{aligned}
$$

From Equations (1) and (39), we get

$$
P_{1,0}=\frac{\lambda z^{*}}{\bar{\lambda} \eta} P_{0,0}
$$

Equation (40) and (4) becomes

$$
\begin{aligned}
& P_{0, j}=B^{j} P_{0,0}, j=1,2, \cdots K-1 \\
& P_{1, j}=B^{j} \frac{\lambda z^{*}}{\bar{\lambda} \eta} P_{0,0}, j=1,2, \cdots K-1,
\end{aligned}
$$

Where $B=\frac{\phi\left(\bar{\lambda}+\lambda z^{*}\right)}{\phi+\lambda\left(1-z^{*}\right) \bar{\phi}}$.

Simplifying Equation (42),

$$
\sum_{j=0}^{K-1} P_{0, j}=\frac{1-B^{K}}{1-B} P_{0,0}
$$

The probability $P_{0, K}$ that the server is idle is calculated by using Equations (7), (42), (43) and (39) as follows

$$
P_{0, K}=\frac{\bar{\lambda} \mu}{\lambda} B^{K} P_{1, K}
$$


From Equations (35), (44) and (45), we obtain

$$
P_{1, K}=\frac{\phi(\mu-\lambda) \lambda\left(1-z^{*}\right)}{\bar{\lambda} \mu\left[(\mu-\eta) \lambda\left(1-z^{*}\right) \bar{\phi}\left(1-B^{K}\right)+\phi \mu\left(1-B^{K} z^{*}\right)\right]}
$$

Substitute above Equation in (39) and (45),

$$
\begin{aligned}
& P_{0,0}=\frac{\phi(\mu-\lambda)(1-B)}{(\mu-\eta) \lambda\left(1-z^{*}\right) \bar{\phi}\left(1-B^{K}\right)+\phi \mu\left(1-B^{K} z^{*}\right)} . \\
& P_{0, K}=\frac{\phi(\mu-\lambda)\left(1-z^{*}\right) B^{K}}{(\mu-\eta) \lambda\left(1-z^{*}\right) \bar{\phi}\left(1-B^{K}\right)+\phi \mu\left(1-B^{K} z^{*}\right)}
\end{aligned}
$$

Using Equation (47) in (44),

$$
\sum_{j=0}^{K-1} P_{0, j}=\frac{\phi(\mu-\lambda)\left(1-B^{K}\right)}{(\mu-\eta) \lambda\left(1-z^{*}\right) \bar{\phi}\left(1-B^{K}\right)+\phi \mu\left(1-B^{K} z^{*}\right)}
$$

Using Equation (49) in (34), we get

$$
\sum_{j=0}^{K-1} G_{j}(1)=\frac{\left(\phi+\lambda\left(1-z^{*}\right) \bar{\phi}\right)(\mu-\lambda)\left(1-B^{K}\right)}{(\mu-\eta) \lambda\left(1-z^{*}\right) \bar{\phi}\left(1-B^{K}\right)+\phi \mu\left(1-B^{K} z^{*}\right)}
$$

Therefore, Equation (30) or (37 can be framed as

$$
\mathrm{E}\left[\mathrm{L}_{\mathrm{Wv}}\right]=\frac{\bar{\phi}}{\phi} \frac{\left(\phi »+(»-\eta) »\left(1-\mathrm{z}^{*}\right) \bar{\phi}\right)(1 / 4-»)\left(1-\mathrm{B}^{\mathrm{K}}\right)}{(1 / 4-\eta) »\left(1-\mathrm{z}^{*}\right) \bar{\phi}\left(1-\mathrm{B}^{\mathrm{K}}\right)+\phi^{1 / 4}\left(1-\mathrm{B}^{\mathrm{K}} \mathrm{z}^{*}\right)}
$$

Now, we derive the mean system size when the server is in the regular busy period, denoted by $E\left[L_{K}\right]$. From Equation (25), using L'Hospital rule, we obtain 


$$
\begin{aligned}
E\left[L_{K}\right]= & G_{K}^{\prime}(1)=\frac{\phi}{2(\mu-\lambda)} \sum_{j=0}^{K-1} G_{j}^{\prime \prime}(1)+\frac{\phi\left(\mu+\eta(\lambda-\mu)-\lambda^{2}\right)}{(\mu-\lambda)^{2}} \sum_{j=0}^{K-1} G_{j}^{\prime}(1)+\frac{\phi \lambda \bar{\lambda}(\mu-\eta)}{(\mu-\lambda)^{2}} \sum_{j=0}^{K-1} G_{j}(1) \\
& +\frac{\lambda \bar{\lambda} \mu}{(\mu-\lambda)^{2}} P_{0, K}+\frac{\phi \lambda \bar{\lambda} \eta}{(\mu-\lambda)^{2}} \sum_{j=0}^{K-1} P_{0, j}
\end{aligned}
$$

where $G_{j}^{\prime \prime}(1)$ is obtained by differentiating twice $G_{j}(z)$ at $z=1$ for $j=0,1, \cdots K-1$. Differentiating twice (19) and (20) and substituting $z=1$ gives

$$
G_{j}^{\prime \prime}(1)=\frac{2}{\phi}\left[\bar{\phi} \lambda\left(\bar{\eta} G_{j}(1)+\eta P_{0, j}\right)+(\bar{\phi}(\lambda+\bar{\eta})-1) G_{j}^{\prime}(1)\right], j=0,1, \cdots K-1
$$

Substituting Equation (53) in (52), we obtain

$$
\begin{aligned}
& E\left[L_{K}\right]=\frac{(\phi \lambda \bar{\mu}+(\lambda-\eta)(\mu-\lambda))}{(\mu-\lambda)^{2}} E\left[L_{W V}\right]+\frac{\phi \lambda \eta \bar{\mu}+\lambda \eta(\mu-\lambda)}{(\mu-\lambda)^{2}} \sum_{j=0}^{K-1} P_{0, j} \\
& +\frac{\phi \lambda \bar{\mu}(\lambda-\zeta)+\lambda \bar{\eta}(\mu-\lambda)}{(\mu-\lambda)^{2}} \sum_{j=0}^{K-1} G_{j}(1)+\frac{\lambda \bar{\lambda} \mu}{(\mu-\lambda)^{2}} P_{0, K}
\end{aligned}
$$

where $E\left[L_{W V}\right]$ is calculated by Equation (51) and the probability when the server is idle $\left(P_{0, K}\right)$, $\sum_{j=0}^{K-1} P_{0, j}$ and $\sum_{j=0}^{K-1} G_{j}(1)$ are calculated by using Equations (48), (49) and (50). Since L be the number of customers in the system. The mean system size $E[L]=E\left[L_{W V}\right]+E\left[L_{K}\right]$ can be calculated from Equations (51) and (54).

P. Vijaya Laxmi is an Associate Professor in the Department of Applied Mathematics, Andhra University, Visakhapatnam, India. She received her M.Sc and Ph.D. degrees from Indian Institute of Technology, Kharagpur, India. Her main areas of research interest are continuous and discrete-time queueing models and their applications. She has publications in various journals like "Operations Research Letters," "Queueing Systems," "Applied Mathematical Modelling," "International Journal of Applied Decision Sciences," "Journal of Industrial and Management Optimization (JIMO)," etc.

Dr. P. Rajesh is working as an Associate Professor, Department of Mathematics, Vignan's Institute of Information Technology, Visakhapatnam, India. He received his M.Sc and Ph.D degrees from Andhra University, and M.phil degree from Sri Venkateswara University, India. His research interests are Operations Research, Stochastic Modelling and Queueing Theory. He has publications in various national and international Journals. 